Grafting chimeric antigen receptors to redirect $\mathrm{T}$ cells

\section{Carving the CAR}

SR Riddell and U Protzer

Gene Therapy (2010) 17, 1191-1192; doi:10.1038/gt.2010.96; published online 29 July 2010

Chimeric antigen receptors (CARs) are major histocompatibility complex (MHC)-independent one-polypeptide chain receptor molecules that, when expressed in T cells, can redirect specificity towards tumor or viral antigens. The antigen-binding domain of the CAR is derived from a single-chain antibody fragment and the transmembrane and signalling domains from the $\mathrm{T}$-cell receptor CD3 $\zeta$ chain, and optionally includes a costimulatory molecule such as CD28. CARs therefore combine the antigen specificity of an antibody and the property to activate a cytotoxic $\mathrm{T}$ cell in a single fusion molecule. A spacer is required between the extracellular antigen-binding domain and the transmembrane moiety. Most commonly, the constant immunoglobulin G (IgG)1 hinge$\mathrm{CH} 2-\mathrm{CH} 3 \mathrm{Fc}$ domain is used as a spacer domain, resulting in dimerization and thereby increasing CAR expression on the cell surface.

In this issue, Hombach et al. ${ }^{1}$ reveal that the IgG1 spacer used in most CARs may also bind to surface IgG Fc gamma receptors expressed on macrophages and natural killer cells. This binding activates both the engineered $\mathrm{T}$ cells and the innate immune cells independent of the specificity of the CAR binding domain. Activation of CAR-positive $\mathrm{T}$ cells and $\mathrm{FC}_{\mathrm{C}}$ receptor-bearing cells through Fc $\gamma \mathrm{R}$ binding led to an unwanted, 'off-target' pro-inflammatory immune response. Hombach et al. ${ }^{1}$ provide a solution to this problem by modifying the sequences required for $\mathrm{Fc}$ binding in the constant immunoreceptor $\mathrm{Fc}$ domain, which eliminated interaction with Fc-positive cells but preserved stable expression of CARs on the surface of T cells and specific antigen recognition.

In recent years, a variety of CARs with different $\mathrm{SCFv}_{\mathrm{V}}$-binding domains have been designed. ${ }^{2}$ These CARs possess binding specificities for tumor-associated antigens, such as carcinoembryonic antigen and melanoma-associated antigens, CD30 and CD19, or for virus-infected cells, such as HIV or hepatitis B virus. ${ }^{3}$ Clinical trials that use CAR-modified $\mathrm{T}$ cells for adoptive immunotherapy of malignant diseases have been implemented and additional trials are planned. Owing to its one-polypeptide chain design and modular composition, the use of CARs substantially simplifies redirecting T cells with defined specificity. Upon antigen engagement, the intracellular signalling domain initiates T-cell activation, resulting in T-cell proliferation, secretion of pro-inflammatory cytokines and cytolysis of the antigen-positive target cell. There are major differences between the natural $\mathrm{T}$ cell receptor and a CAR, which confer potential advantages and disadvantages. CAR recognition is independent from antigen presentation on MHC, and allows broad clinical application for patients of any HLA haplotype. The lack of MHC restriction may also allow effective therapy of tumors that escape MHC-restricted $\mathrm{T}$ cells by downregulating $\mathrm{MHC}$ expression or through defects in antigen processing. However, early versions of CARs often provided weak activation of engineered $\mathrm{T}$ cells, mainly because CAR expression was unstable and costimulatory molecules were not, or only inadequately, engaged. The modular nature of CARs has enabled researchers to modify the CAR structure to achieve better activity. In the early research on CARs, it became obvious that inserting a 'spacer' domain, the IgG1 Fc being most commonly used, between the antigen-binding and transmembrane moieties improved expression and binding. ${ }^{4}$ Later on, research focussed on improving the signalling capacity by fusing costimulatory domains, such as CD28, OX40, $4-1 B B$ or the src family kinase lck,

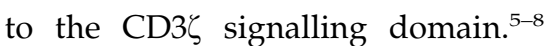
$\mathrm{T}$ cells carrying CARs with $\mathrm{CD} 3 \zeta$ and CD28 signalling domains proved to be superior in vivo and were able to reject established tumors in mice. ${ }^{9}$ Using $\mathrm{T}$ cells with natural specificity for Epstein-Barr virus is another strategy to provide appropriate costimulation through intermittent T-cell receptor ligation, and improved the persistence and probably also the activity of CARgrafted $\mathrm{T}$ cells in tumor patients. ${ }^{10}$

Severe side effects reported from recent clinical trials of adoptive immunotherapy with CAR-redirected $\mathrm{T}$ cells are a poignant reminder that much remains to be learned to realize the therapeutic promise of this approach. In one case, reactivity of the CAR antibody-binding domain with normal antigen-expressing cells was implicated as the cause of a cytokine storm and organ toxicity, ${ }^{11}$ and an unexplained sepsis-like syndrome occurred in a second case. ${ }^{12}$

The work of Hombach et al. ${ }^{1}$ illustrates the potential for 'offtarget' activation of engineered $\mathrm{T}$ cells via $\mathrm{FCR}$ binding to induce unwanted cytokine release and tissue damage-and, importantly, provides an improvement in CAR design that may help to avoid these side effects. It is anticipated that further improvements in CAR design will focus on increasing the specificity of the CARredirected T-cell response and minimizing the inflammatory response that is elicited. This should improve the safety and efficacy of adoptive immunotherapy with CAR-engineered $\mathrm{T}$ cells.

\section{Conflict of interest}

The authors declare no conflict of interest.

Dr SR Riddell is at D3-100, Fred Hutchinson Cancer Research Center, 1100 Fairview Avenue N., Seattle, WA 98109, USA. Dr U Protzer is at the Institute of Virology, Technische Universität München/Helmholtz Zentrum München, Troger Str. 30, 81675 München, Germany.

E-mail: protzer@tum.de

1 Hombach A, Hombach AA, Abken $\mathrm{H}$. Adoptive immunotherapy with genetically engineered $T$ cells: modification of the IgG1 $\mathrm{Fc}_{\mathrm{c}}$ 'spacer' domain in the extracellular moiety of chimeric antigen receptors avoids 'off-target' activation 
and unintended initiation of an innate immune response. Gene Therapy 2010; 17: 1206-1213.

2 Gross G, Waks T, Eshhar Z. Expression of immunoglobulin-T-cell receptor chimeric molecules as functional receptors with antibody-type specificity. Proc Natl Acad Sci USA 1989; 86: 10024-10028.

3 Bohne F, Chmielewski M, Ebert G, Wiegmann K, Kurschner T, Schulze A et al. T cells redirected against hepatitis B virus surface proteins eliminate infected hepatocytes. Gastroenterology 2008; 134: 239-247.

4 Moritz D, Groner B. A spacer region between the single chain antibody- and the CD3 zeta-chain domain of chimeric T cell receptor components is required for efficient ligand binding and signaling activity. Gene Ther 1995; 2: 539-546.

5 Hombach A, Sent D, Schneider C, Heuser C, Koch D, Pohl C et al. T-cell activation by recombinant receptors: CD28 costimulation is required for interleukin 2 secretion and receptormediated T-cell proliferation but does not affect receptor-mediated target cell lysis. Cancer Res 2001; 61: 1976-1982.

6 Geiger TL, Nguyen P, Leitenberg D, Flavell RA. Integrated src kinase and costimulatory activity enhances signal transduction through single-chain chimeric receptors in $\mathrm{T}$ lymphocytes. Blood 2001; 98: 2364-2371.

7 Beecham EJ, Ma Q, Ripley R, Junghans RP. Coupling CD28 co-stimulation to immunoglobulin T-cell receptor molecules: the dynamics of T-cell proliferation and death. J Immunother 2000; 23: 631-642.

8 Maher J, Brentjens RJ, Gunset G, Rivière I, Sadelain M. Human T-lymphocyte cytotoxicity and proliferation directed by a single chimeric TCRzeta/ CD28 receptor. Nat Biotechnol 2002; 20: 70-75.

9 Haynes NM, Trapani JA, Teng MW, Jackson JT, Cerruti L, Jane SM et al.
Single-chain antigen recognition receptors that costimulate potent rejection of established experimental tumors. Blood 2002; 100: 3155-3163.

10 Pule MA, Savoldo B, Myers GD, Rossig C, Russell HV, Dotti G et al. Virus-specific $\mathrm{T}$ cells engineered to coexpress tumor-specific receptors: persistence and antitumor activity in individuals with neuroblastoma. Nat Med 2008; 14: 1264-1270.

11 Morgan RA, Yang JC, Kitano M, Dudley $\mathrm{ME}$, Laurencot CM, Rosenberg S. Case report of a serious adverse event following the administration of $\mathrm{T}$ cells transduced with a chimeric antigen receptor recognizing ERBB2. Mol Ther 2010; 18: 843-851.

12 Brentjens R, Yeh R, Bernal Y, Riviere I, Sadelain M. Treatment of chronic lymphocytic leukemia with genetically targeted autologous T cells: case report of an unforeseen adverse event in a phase I clinical trial. Mol Ther 2010; 18: 666-668. 